\section{JTI}

JOURNAL OF

TRAUMA AND INJURY

\title{
Management of a Retained Bullet in the Corpora Cavernosa after a Civilian Gunshot Injury: A Rare Scenario
}

\author{
Ali Abdel Raheem, M.D., Ph.D. ${ }^{1,2}$, Ibrahim Alowidah, M.D. ${ }^{1}$, \\ Mohamed Almousa, M.D. ${ }^{1}$, Mohamed Alturki, M.D. ${ }^{1}$ \\ ${ }^{1}$ Department of Urology, King Saud Medical City, Riyadh, Saudi Arabia \\ ${ }^{2}$ Department of Urology, Tanta University Hospital, Tanta, Egypt
}

Received: March 4, 2020

A 24-year-old man presented to King Saud Medical City emergency department with a retained bullet in his penis following a civilian exchange of gunfire. After an initial assessment, the patient was taken to the operating room. Penile exploration was performed, the bullet was extracted successfully, and the corpora cavernosa were repaired properly. A 6-week follow-up showed full healing with preservation of erectile function. Immediate surgical intervention is mandatory as the primary treatment for penile gunshot injury to ensure proper anatomical and functional recovery.

Keywords: Penis; Gunshot wound; Assessment, outcomes

Correspondence to

Ali Abdel Raheem, M.D., Ph.D. Department of Urology, King Saud Medical City, Ulaishah, Riyadh 12746, Saudi Arabia

Tel: +966-53-253-2128

E-mail:aliraheem82@yahoo.com

\section{INTRODUCTION}

Penile trauma accounts for fewer than $1 \%$ of cases of civilian trauma, and penetrating penile injuries caused by gunshots are extremely rare [1,2]. Here we present an interesting case of a civilian gunshot injury in which the bullet traversed the left thigh, causing a fracture of the femur, and was retained in the corpora cavernosa of the penis. In this report, we highlight important aspects of the optimal management of the patient.

\section{CASE REPORT}

A 24-year-old man presented to the emergency ward with a history of a gunshot wound 4 hours prior to arrival. We started the advanced trauma life support resuscita- 


\section{JTI}

tion protocol. Upon general examination, the patient was vitally stable, complaining of pain in the left thigh and penis. His abdomen was soft and lax, he had no neurological defects, and pulses were observed in all extremities. A local examination revealed the presence of swelling and hematoma in the left thigh with entrance and exit wounds on the posterior lateral and medial aspects of the left thigh. A genital exam showed a clean wound (the bullet entrance) measuring $0.5 \mathrm{~cm}$ on the left side of the penile shaft with no exit wound (Fig. 1). The bullet was palpable. No penile swelling, hematoma, or bleeding through the urethra was present. The scrotal skin and both testes were normal. A computed tomography (CT) scan of the abdomen and pelvis with angiography of both lower limbs was performed to determine the extent and severity of the injury (Fig. 2). CT showed a retained bullet in the distal penile shaft. Ascending urethrography ruled out an associated urethral injury and confirmed the presence of
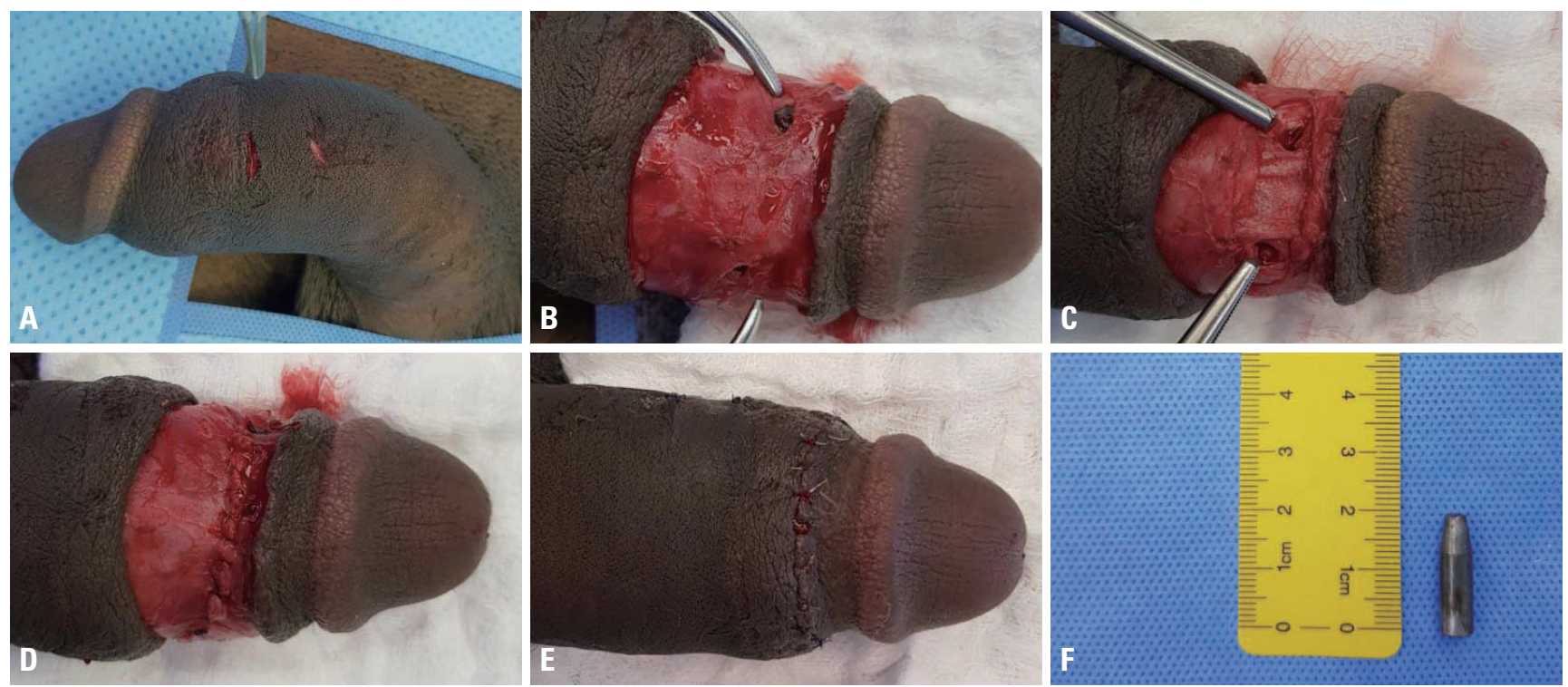

Fig. 1. Summary of the surgical steps. (A) An image taken before surgery shows the bullet entrance wound. (B) After skin degloving, 2 holes in the tunica albuginea were found, encompassing both ends of the bullet. (C) After removal of the bullet with intact nerve structures at 1- and 11-o'clock. (D) After closure of the tunica albuginea and Buck's fascia with 3/0 Vicryl sutures. (E) Final appearance after skin approximation. (F) The 2-cm bullet.
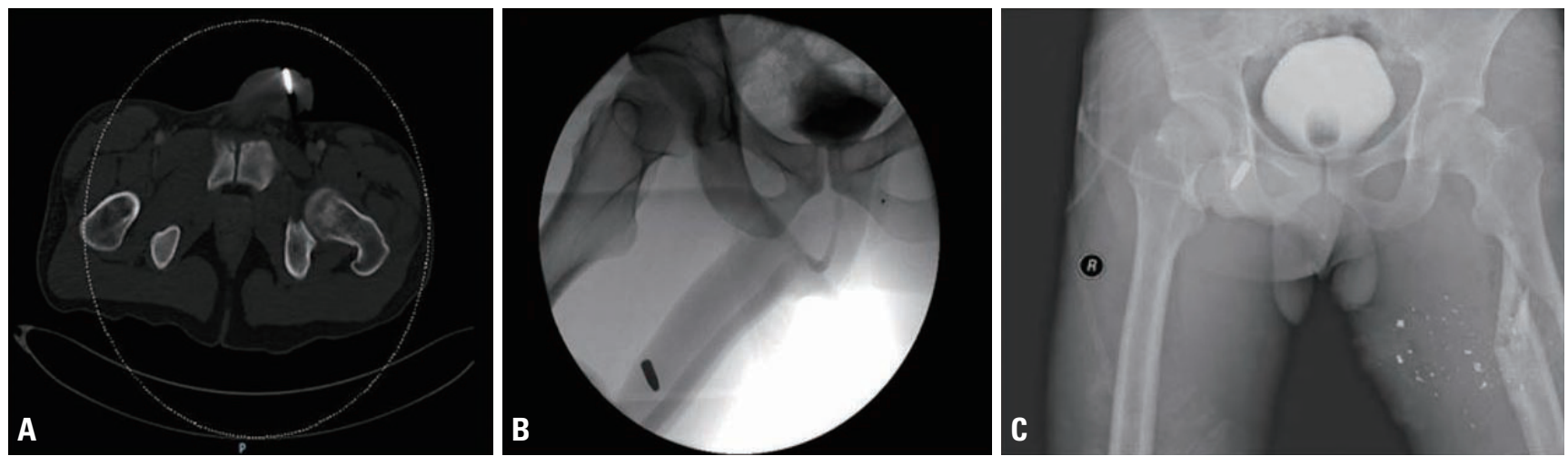

Fig. 2. Imaging studies. (A) A computed tomography scan showed a single non-deformed bullet inside the distal penile shaft. (B) Retrograde urethrography showed an intact urethra with no extravasation, and the bullet was retained inside the corporal tissues. (C) An X-ray of the pelvis and upper limbs showed a left femur fracture. Penile gunshot wounds are often associated with injuries of other organs, the urethra, and/or the corpora cavernosa. 
a single non-deformed bullet in the penis (Fig. 2). After confirmation of the diagnosis, the patient was transferred immediately to the operating room for penile surgical exploration.

\section{Surgical steps}

As shown in Fig. 1, a circumferential subcoronal incision was made with degloving of the penile skin. We identified two holes in the tunica albuginea on the dorsum of the distal penis with both ends of the bullet visible. The bullet was extracted successfully with no intraoperative complications. First, the holes in the cavernous tunica albuginea were sutured with 3/0 Vicryl, and Buck's fascia was then approximated with 3/0 Vicryl over the repaired tunica. An artificial erection test was performed to ensure proper repair. Finally, the skin was sutured with 4/0 Monocryl. A 16-Fr Foley catheter was inserted for 1 day, and after removal the patient had spontaneous micturition. The patient's postoperative course was uneventful. At a 6-week follow-up, the patient reported normal penile erection and ultrasonography of the penis showed no fibrosis or abnormal morphology.

\section{DISCUSSION}

In the present case, we report an extremely rare scenario of a retained bullet in the corpora cavernosa after a civilian gunshot injury. A similar case of a retained bullet was described earlier in the literature. Unlike in our case, the bullet was missed in the primary management, diagnosed after 2 days on X-ray imaging, and treated by exploration and a full-thickness skin graft [3]. This underscores the importance of the initial assessment of gunshot injuries, as they usually involve more than one organ. Performing a CT scan is essential for evaluating the bullet trajectory and identifying ballistic fragments. In our case, the external genitalia were normal except for a small $(<0.5 \mathrm{~cm})$ penile skin wound that, if not properly examined, might have been missed during the primary management [3]. Additionally, a CT scan confirmed presence of the bullet.

Civilian penile gunshot injuries are an extremely rare condition, and up to $90 \%$ of patients had associated other organ injuries, most commonly in the lower limbs (69\%) or scrotum (52\%) [2]. In our case report, an associated left femur fracture was managed successfully by the orthopedic team. A previous study reported that up to $22 \%$ of penetrating penile injuries may be associated with concomitant injury to the urethra [4], and this proportion may be as high as $33 \%$ in cases of gunshot injuries [5]. Of note, the presence of blood at the urethral meatus, hematuria, and/or urinary retention is a warning sign of underlying urethral injury. Therefore, performing retrograde urethrography and/or urethroscopy is mandatory to rule out such injuries. Kunkle et al. [6] studied 63 patients who had gunshot wounds of the penis, and found that associated urethral injuries were present in $17.5 \%$ of these patients, of whom $67 \%$ received primary urethral repair and $33 \%$ underwent suprapubic cystotomy.

In civilian gunshot injuries, minimal tissue destruction is usually present [2]. Interestingly, in the present case, there was a small penile skin wound, with minimal corporal tissue loss $(<2 \mathrm{~cm}$; i.e., a grade 3 penile injury according to the American Association for the Surgery of Trauma classification) [7]. The presence of a corporal injury necessitated immediate penile exploration in our patient. Of note, early surgical exploration and reconstruction of the corpora cavernosa can prevent the development of undesired late complications, such as urethral stricture, penile deformity/curvature, and erectile dysfunction [5]. However, some may advocate nonoperative management of superficial penile skin lesions, contusions, and Buck's fascia lacerations (i.e., grade 1 or 2 penile injuries) [7].

In summary, civilian penile gunshot injuries are a rare entity and usually involve other organs, making a proper initial assessment of the utmost importance to assess the extent of the injury. Underlying urethral involvement may be present in up to one-third of cases, and in such situations, an additional therapeutic option is usually needed. The presence of corporal tissue injury is an absolute indication for immediate penile exploration and reconstruction in order to prevent late complications and to preserve urinary and erectile function.

\section{REFERENCES}

1. Kim JH, Park JY, Song YS. Traumatic penile injury: from 
circumcision injury to penile amputation. Biomed Res Int 2014;2014:375285.

2. Cerwinka WH, Block NL. Civilian gunshot injuries of the penis: the Miami experience. Urology 2009;73:877-80.

3. Shirol SS, Nerli R, Prabha V, Nimbaragi G. Bullet injury to the penis with retained bullet in the penis. Int J Pharm Med \& Bio Sci 2012;1:33-6.

4. Cline KJ, Mata JA, Venable DD, Eastham JA. Penetrating trauma to the male external genitalia. J Trauma 1998;44:492-4.

5. Cavalcanti AG, Krambeck R, Araujo A, Manes CH, Favorito LA.
Penile lesion from gunshot wound: a 43-case experience. Int Braz J Urol 2006;32:56-63.

6. Kunkle DA, Lebed BD, Mydlo JH, Pontari MA. Evaluation and management of gunshot wounds of the penis: 20-year experience at an urban trauma center. J Trauma 2008;64:1038-42.

7. Mohr AM, Pham AM, Lavery RF, Sifri Z, Bargman V, Livingston DH. Management of trauma to the male external genitalia: the usefulness of American Association for the Surgery of Trauma Organ Injury Scales. J Urol 2003;170:2311-5. 